\title{
Induction of protective immunity to malaria
}

\author{
R F ANDERS, G V BROWN, R L COPPEL \& D J KEMP \\ The Walter \& Eliza Hall Institute of Medical Research, Melbourne, \\ Victoria, Australia
}

\begin{abstract}
Introduction
The relative importance of different immune effector mechanisms in human malaria is not clear although antibodies have been clearly implicated as having a role in controlling parasitaemias (1). Recurrent infection with malaria parasites results in high levels of antibodies directed against a great variety of asexual blood-stage antigens (2). Many of these antibodies are probably irrelevant to protection, and identification of those critical antigens which are targets of protective immune responses is an important part of current research directed towards the development of a vaccine against malaria.

Assessment of whether a particular antigen is a target of protective immune responses depends on the availability of purified antigens and/or monospecific antibodies to those antigens. In our work with Plasmodium falciparum we have attempted to clone and express in Escherichia coli as many as possible of the antigens of the asexual blood-stages of $P$. falciparum (3-5). These antigens, produced in $E$. coli, have been used to produce antisera with which the corresponding parasite antigens have been identified and characterized using the strategies outlined below. Fragments of one of these antigens, produced in $E$. coli as $\beta$-galactosidase fusion polypeptides, have been tested for their ability to immunize Aotus monkeys against infection with $P$. falciparum (6). This vaccine trial demonstrated that the ring-infected erythrocyte surface antigen (RESA) could induce protective immune responses and that protection correlated with antibody responses to either of two different repetitive sequences in RESA.
\end{abstract}

\section{Cloning and expression of asexual blood-stage antigens in $E$. coli}

In order to produce individual antigens, total mRNA was isolated from strains of $P$. falciparum growing asynchronously in vitro and the complementary DNA was cloned into $\lambda$ gtl l-Amp3 (3). As the primary screen for clones expressing $P$. falciparum antigens, antibodies from the serum of adult coastal New Guineans were reacted with the recombinants using in situ colony immunoassays. In this way a large number of clones that correspond to many natural immunogens of $P$. falciparum were selected (4, 7). About $20 \%$ of these clones produce the cloned antigen as an abundant $\beta$-galactosidase fused polypeptide. In the remaining clones the parasite antigen most commonly was expressed as a fused polypeptide that was rapidly degraded by bacterial proteases. However, in some instances, for example where the cDNA insert contained a complete coding sequence, low amounts of the parasite antigens were synthesized in a form not associated with $\beta$-galactosidase $(4,8)$. 
mRNA from $P$. falciparum growing asynchronously in vitro<smiles>C=C[C@@H](C(C)CC)[C@H](C)C(=O)O</smiles>

Cloned in $\lambda \mathrm{gt} 10$ then $\lambda \mathrm{gtl} 1 \mathrm{l}-\mathrm{Amp} 3$<smiles>CCCC</smiles>

Selection of antigen-expressing recombinants with human anti-malarial antibodies<smiles>C=[V]</smiles>

Production of monospecific antisera

a. Heterologous antisera

b. Monoclonal antibodies

c. Affinity-purified human antibodies

Identification of corresponding $P$. falciparum antigens

a. Immunoblotting and immunoprecipitation

b. Immunofluorescence microscopy

c. Immunoelectron microscopy

Figure 1. Strategy adopted for cloning natural immunogens of the asexual blood-stages of $\mathrm{P}$. falciparum.

\section{Identification of $\boldsymbol{P}$. falciparum antigens corresponding to cloned antigens}

Antibodies to individual cloned antigens have been used to identify the corresponding parasite antigens. These antibodies were prepared by immunizing rabbits or mice with the expression products or with synthetic peptides corresponding to sequences deduced from the nucleotide sequences of various clones $(9,11)$. An alternative procedure that we have found very useful has been to affinity-purify antibodies from plasma obtained from adults living in areas where malaria is endemic $(8,12)$. The adsorbents used for purifying these human antibodies were prepared by coupling total $E$. coli lysates to CNBr-activated Sepharose, a procedure that has enabled us to obtain antibodies to cloned antigens that were not necessarily expressed as abundant fused polypeptides (13). The specificity of these affinity-purified human antibodies was demonstrated by their reactivity in colony immunoassays with a very restricted set of clones when compared with the starting plasma pool. In fact it was frequently possible to obtain purified antibody preparations that reacted strongly with clones that were not readily detected by the starting plasma pool (13). Immunoblotting experiments with these purified antibodies have enabled us to identify more than 30 different asexual bloodstage antigens that are represented in our library of antigen-expressing clones. However, in some cases the antibodies prepared using these clones react with more than one parasite antigen. Although this may reflect proteolytic cleavage of some parasite antigens it may also reflect cross-reactivities amongst these different proteins (see below). 


\section{Possible targets of protective immune responses}

In vivo laboratory experimentation with $P$. falciparum is restricted to Aotus and Saimiri monkeys. Because of this it is not possible to test directly in vivo the many $P$. falciparum antigens that are now available. Therefore, it is necessary to use less direct evidence to assess the possible involvement of various antigens as targets of immune effector mechanisms and as potential vaccine components.

The ultrastructural location of various antigens is one characteristic that has been used to identify antigens that may be targets of protective immune responses. Of particular interest are antigens that are either on the surface of the merozoite, associated with the membrane of the infected red cell or in micronemes or rhoptries, apical organelles in the merozoite which discharge their contents at the time of merozoite invasion. Antigens in these locations are assumed to be accessible to the host immune response. The techniques of immunofluorescence microscopy and particularly immunoelectron microscopy using the protein A-gold technique have been used to identify a number of antigens in these locations (Table 1).

A second important approach to identifying target antigens has employed antibodies that have been demonstrated to inhibit the growth of $P$. falciparum in vitro. Although this approach has been successfully followed using monoclonal antibodies $(22,24)$ or human antibodies affinity purified either on parasitized cells (17) or synthetic peptides (25) we have not obtained useful data using antibodies raised against, or affinity purified on, $P$. falciparum antigens expressed in E. coli.

Table 1. P. falciparum antigens that may be targets of protective immune responses.

\begin{tabular}{|c|c|}
\hline Location & Antigen \\
\hline $\begin{array}{l}\text { Merozoite surface } \\
\text { antigens }\end{array}$ & $\begin{array}{l}\text { Merozoite surface protein precursor }(14) \\
\text { Glycophorin binding proteins }(15,16)\end{array}$ \\
\hline $\begin{array}{l}\text { Antigens associated with } \\
\text { the membrane of the } \\
\text { infected erythrocyte }\end{array}$ & $\begin{array}{l}\text { Ring-infected erythrocyte surface antigen }(6,17) \\
\text { Mature-infected erythrocyte surface antigen (18) } \\
\text { Cytoadherence molecule (19) }\end{array}$ \\
\hline Rhoptry antigens & $\begin{array}{ll}\text { Pf140 } & (20) \\
\text { Pf41 } & (21) \\
\text { Ag44 } & (5) \\
\text { QF3 } & (22)\end{array}$ \\
\hline
\end{tabular}

\section{The ring-infected erythrocyte surface antigen (RESA)}

RESA is one asexual blood-stage antigen that has been identified as a target of antibodies with anti-parasitic activity and this antigen is currently being assessed as a candidate vaccine molecule. Immunoelectron microscopy using antisera raised against fragments of the RESA molecule expressed in $E$. coli has been used to locate RESA both within micronemes in schizonts and free merozoites and also associated with the membrane of ring-infected erythrocytes (26). It is probable that RESA is discharged from the merozoite apical pore, possibly via the rhoptries, at the time of parasite invasion. When associated with the red cell membrane RESA is largely insoluble in solutions of the non-ionic detergent Triton X-100 (26). 
This suggests that RESA is anchored at the red cell membrane through an interaction with the membrane skeleton. RESA has an apparent molecular mass (Mr) of 155,000 determined by mobility on sodium dodecyl sulphate (SDS) polyacrylamide gel electrophoresis (10). An antigen (Pf155) of the same size and in the same membrane location was detected with human anti-malarial antibodies eluted from the surface of glutaraldehyde-fixed and air dried monolayers of infected cells (27). These antibodies were potent inhibitors of merozoite invasion in vitro (17). RESA and Pf155 are clearly the same molecule and, although a number of antisera and monoclonal antibodies raised against fragments of RESA failed to block merozoite invasion (unpublished results), RESA was considered to be a prime candidate for testing in vaccine trials in monkeys.

The complete structure of the RESA gene has been determined from the sequence of genomic clones (Favaloro et al. submitted for publication). The gene contains one short intron which separates a short exon 1, encoding the N-terminal 65 amino acids, from a very much larger exon 2 encoding the rest of the molecule. RESA like most other malaria antigens that have been sequenced, contains short sequence repeats. In RESA there are two blocks of repeats, both in exon 2, one located at the C-terminal end of the molecule (3' repeat) and the other near the middle of the polypeptide chain (5' repeat) (28). The 3' repeat includes five copies of an eight amino acid sequence Glu Glu Asn Val Glu His Asp Ala followed by 28 copies of the four amino acid sequence Glu Glu Asn Val occasionally interspersed by related tetrameric or trimeric sequences. The 5' repeat is more degenerate, containing two copies of the 11 amino acid sequence Asp Asp Glu His Val Glu Glu Pro Thr Val Ala surrounded by five other sequences derived from this 11-mer by deletions or conservative substitutions. As found in the circumsporozoite (CS) protein (29) and the S-antigen $(9,30)$, two other malaria antigens that have been studied in detail, the repeats of RESA appear to be the immunodominant parts of the molecule. Studies with synthetic peptides have shown that all three repeating sequences (4-mer, 8-mer and 11-mer) encode antigenic epitopes that induce antibody responses in individuals exposed to recurrent infections with $P$. falciparum (unpublished results).

Table 2. Recombinant polypeptides corresponding to fragments of RESA used in an Aotus monkey vaccine trial.

\begin{tabular}{lll}
\hline Group & Immunogen & Source of Immunogen \\
\hline 1 & FP Ag28 & 3' repeat of RESA (isolate FCQ27/PNG) \\
2 & FP Ag632 & 5' repeat of RESA (isolate NF7) \\
3 & FP Ag631 + & Non-repeat sequences on either side of the 5' repeat of \\
& FP Ag633 & RESA (isolate FN7) but also including the 5' repeat \\
4 & FP TA10 & $\begin{array}{l}\text { A fused polypeptide containing uncharacterized sequences } \\
\text { of a taeniid protein }\end{array}$ \\
\hline
\end{tabular}

Fused polypeptides corresponding to different regions of the RESA polypeptide were tested in Aotus monkeys for their ability to confer protection against overwhelming infection with $P$. falciparum (Table 2) (6). Animals were immunized with fused polypeptides in complete Freund's adjuvant, boosted six weeks later with the same amount of antigen in a mixture of complete and incomplete Freund's adjuvant (1:10, v:v) and challenged two weeks la- 
ter with an intravenous injection of $1 \times 10^{6}$ Aotus red cells infected with the Indochina I/CDC strain of $P$. falciparum. Parasitaemias were monitored by daily examination of Giemsastained blood films. Animals in which parasitaemias reached $10 \%$ were given chemotherapy, while those which survived with parasitaemias not reaching $10 \%$ were regarded as resistant.

Table 3. Effect of immunization with RESA

on course of parasitaemia in Aotus monkeys challenged with P. falciparum.

\begin{tabular}{lccc}
\hline \multirow{2}{*}{ Group } & Animal No. & \multicolumn{2}{c}{ Maximum parasitaemia } \\
& & \% RBCs infected & Day \\
\hline 1 (Ag28) & Ao-421 & 0.4 & 19 \\
& Ao-420 & 1.9 & 15 \\
& Ao-423 & 10.2 & 11 \\
& Ao-424 & 16.0 & 16 \\
& Ao-430 & 19.0 & 14 \\
& & & 19 \\
& Ao-426 & 1.7 & 30 \\
& Ao-412 & 1.8 & 27 \\
& Ao-427 & 2.2 & 19 \\
& Ao-422 & 3.7 & 28 \\
& & & 16 \\
& Ao-417 & 0.08 & 11 \\
& Ao-416 & 2.8 & 20 \\
& Ao-428 & 3.6 & 14 \\
& Ao-425 & 7.5 & 30 \\
& Ao-407 & 10.2 & 30 \\
& & & 11 \\
\hline
\end{tabular}

Nine of 14 immunized animals exhibited some degree of resistance to the challenge infection whereas all control animals that were challenged were susceptible (Table 3). This evidence that RESA could induce protective immunity was reinforced by the results of serologic analyses in which the antibody responses of individual monkeys against peptides corresponding to the three major repetitive sequences in RESA were measured (Table 4). Of the five animals immunized with a fused polypeptide largely comprised of the 3 ' repeats of RESA (Group 1) the three animals that were susceptible had low antibody titres to the eight amino acid repetitive sequence whereas the two resistant animals had high antibody titres to this sequence. All five animals had high antibody titres to the repetitive four amino acid sequence. Of nine monkeys immunized with fused polypeptides which included 5 ' repeat sequences (Groups 2 \& 3), seven were resistant. All seven resistant monkeys had high titre antibody responses to the 11 amino acid repetitive sequence contained within the 5' repeat whereas the two susceptible monkeys had very low antibody titres to this sequence. Thus antibody responses induced by immunization, and measured prior to challenge, against two dif- 
ferent repetitive sequences (the 5' repeat 11-mer and the 3' repeat 8-mer) in RESA predicted which animals were rendered resistant to overwhelming infection with P. falciparum. It is of interest that neither antibody responses against the dominant 4-mer repetitive sequences nor anti-RESA antibodies measured by immunofluoresence titre on glutaraldehyde fixed and air dried films predicted which monkeys would be resistant (6).

Table 4. Antibody responses to RESA repetitive sequences in immunized Aotus monkeys.

\begin{tabular}{lcccc}
\hline Group & Antimal No. & ELISA-1 & ELISA-2 & ELISA-3 \\
\hline 1 (Ag28) & Ao-421 R & $<0.1$ & 2.0 & 1.9 \\
& Ao-420 R & $<0.1$ & 1.5 & 1.8 \\
Ao-423 S & $<0.1$ & $<0.1$ & 2.1 \\
Ao-424 S & $<0.1$ & $<0.1$ & 2.1 \\
& Ao-430 S & $<0.1$ & 0.2 & 1.8 \\
& & & & $<0.1$ \\
$2($ Ag632) & Ao-426 R & 1.8 & $<0.1$ & 0.1 \\
& Ao-412 R & 1.9 & $<0.1$ & $<0.1$ \\
& Ao-427 R & 2.0 & $<0.1$ & $<0.1$ \\
& Ao-422 R & 1.9 & $<0.1$ & $<0.1$ \\
$3($ Ag631 + Ag633) & Ao-417 R & 1.8 & $<0.1$ & $<0.1$ \\
& Ao-416 R & 1.4 & $<0.1$ & $<0.1$ \\
& Ao-428 R & 1.9 & $<0.1$ & $<0.1$ \\
& Ao-425 S & $<0.1$ & $<0.1$ & $<0.1$ \\
& Ao-407 S & 0.2 & $<0.1$ & $<0.1$ \\
& Ao-409 S & $<0.1$ & $<0.1$ & $<0.1$ \\
\hline Ao-411 S & $<0.1$ & $<0.1$ & $<0.1$ &
\end{tabular}

* Antibody responses were measured on 1:2,000 dilutions of monkey sera by ELISA using three different peptides corresponding to repetitive sequences in RESA: ELISA-1, 5' repeat 11-mer (DDEHVEEPTVA) x 2; ELISA-2, 3' repeat 8-mer (EENVEHDA); ELISA-3, 3 ' repeat 4-mer $(E E N V) \times 4$. The optical densities at $414_{\mathrm{nm}}$ for three replicates were averaged.

$\mathrm{R}$ and $\mathrm{S}$ indicate whether the monkey was resistant or susceptible.

\section{Discussion}

RESA is one of several different antigens in the asexual blood stages of $P$. faciparum that have now been implicated as targets of protective immune responses (Table 1). Increasingly, it seems likely that the development of naturally acquired immunity in malaria reflects the combined activity of antibodies and/or T cells with specificity for a number of different antigens including not only several asexual stage antigens but also the circumsporozoite protein and perhaps antigens of the exoerythrocytic stages in the liver as well. This contrasts markedly to immunity in some other parasitic protozoa, in particular, the African trypanosome, where immunity is largely directed against a single antigen, the variable surface glycopro- 
tein. The multiplicity of target antigens in $P$. falciparum offers the possibility that a multicomponent malaria vaccine will be developed. Such a vaccine should decrease the likelihood that the benefits of immunization will be circumvented by the emergence of new antigenic variants of $P$. falciparum. In addition it may be possible to include, in the one vaccine, antigens expressed on the surface of $P$. falciparum gametes or the developing zygote, antibodies to which have been shown to block transmission of malaria by the mosquito host (31).

The unusual repeat structures in malaria antigens has allowed rapid identification of naturally immunogenic epitopes in these proteins. The results of the RESA vaccine trial in Aotus monkeys and the studies on CS proteins indicate that the epitopes encoded by some repetitive sequences are targets of protective immune responses. However, given the abundance of antibodies to some repetitive sequences in some non-immune individuals or immunized animals much of the antibody response to repeats is probably irrelevant to protection. Recently we have discovered that the epitopes encoded by repetitive sequences in antigens of $P$. falciparum are involved in multiple networks of cross-reactivities (32). We believe that these cross-reactivities provide a mechanism whereby the repeat structures in these antigens favour parasite survival possibly because they delay the development of high-affinity antibody responses to critical epitopes (32). Thus for a malaria vaccine to be optimally effective it may be necessary not only to deliver the critical epitopes in a suitably immunogenic form but also in isolation from the many cross-reacting epitopes.

Finally, studies on the very variable $\mathrm{S}$-antigen sequences suggest that for at least some antigens, the sequence of the repeats and hence the antigenic specificity can rapidly change (5). Although the repeats in both RESA and the CS proteins of $P$. falciparum seem to be highly conserved, it is of some concern that a vaccine composed of these repeats may rapidly select variants that evade the immune responses induced by immunization.

\section{References}

1 Cohen S, McGregor IA, Carrington S. Gamma-globulin and acquired immunity to human malaria. Nature, 1961, 192, 733-737.

2 Brown GV, Anders RF, Stace JD, Alpers MP, Mitchell GF. Immunoprecipitation of biosynthetically-labelled proteins from different Papua New Guinea Plasmodium falciparum isolates by sera from individuals in the endemic area. Parasite Immunol, 1981, 3, 283-298.

3 Kemp DJ, Coppel RL, Cowman AF, Saint RB, Brown GV, Anders RF. Expression of Plasmodium falciparum blood-stage antigens in Escherichia coli: Detection with antibodies from immune humans. Proc Natl Acad Sci USA, 1983, 80, 3787-3791.

4 Anders RF, Coppel RL, Brown GV, Saint RB, Cowman AF, Lingelbach KR, Mitchell GF, Kemp DJ. Plasmodium falciparum complementary DNA clones expressed in Escherichia coli encode many distinct antigens. Molec Biol Med, 1984, 2, 177-192.

5 Kemp DJ, Coppel RL, Stahl HD, Bianco AE, Corcoran LM, McIntyre P, Langford CJ, Favaloro JM, Crewther PE, Brown GV, Mitchell GF, Culvenor JG, Anders RF. Genes for antigens of Plasmodium falciparum. Parasitology, 1986, 91, S83-108.

6 Collins WE, Anders RF, Pappaioanou M, Campbell GH, Brown GV, Kemp DJ, Coppel RL, Skinner JC, Andrysiak PM, Favaloro JM, Corcoran LM, Broderson JR, Mitchell GF, Campbell CC. Protection of Aotus monkeys by immunization with recombinant proteins of the ring-infected erythrocyte surface antigen (RESA) of Plasmodium falciparum. Nature, 1986, 323, 259-262.

7 Stahl HD, Bianco AE, Crewther PE, Anders RF, Kyne AP, Coppel RL, Mitchell GF, Kemp DJ, Brown GV. Sorting large numbers of clones expressing Plasmodium falcipa- 
rum antigens in Escherichia coli by differential antibody screening. Molec Biol Med, 1986, 3, 351-368.

8 Coppel RL, Favaloro JM, Crewther PE, Burkott TR, Bianco AE, Stahl HD, Kemp DJ, Anders RF, Brown GV. A blood-stage antigen of Plasmodium falciparum shares determinants with the sporozoite coat protein. Proc Natl Acad Sci USA, 1985, 82, 5121-5125.

9 Coppel RL, Cowman AF, Lingelbach KR, Brown GV, Saint RB, Kemp DJ, Anders RF. Isolate-specific $\mathrm{S}$-antigen of Plasmodium falciparum contains a repeated sequence of eleven amino acids. Nature, 1983, 306, 751-756.

10 Coppel RL, Cowman AF, Anders RF, Bianco AE, Saint RB, Lingelbach KR, Kemp DJ, Brown GV. Immune sera recognize on erythrocytes a Plasmodium falciparum antigen composed of repeated amino acid sequences. Nature, 1984, 310, 789-791.

11 Anders RF, Shi P-T, Scanlon DB, Leach SJ, Coppel RL, Brown GV, Stahl H-D, Kemp DJ. Antigenic repeat structures in proteins of Plasmodium falciparum. In Ciba Foundation Symposium on Synthetic Peptides as Antigens, Symposium 119, 1985, 164-175.

12 Stahl H-D, Crewther PE, Anders RF, Brown GV, Coppel RL, Bianco AE, Mitchell GF, Kemp DJ. Interspersed blocks of repetitive and charged amino acids in a dominant immunogen of Plasmodium falciparum. Proc Natl Acad Sci USA, 1985, 82, 543-547.

13 Crewther PE, Bianco AE, Brown GV, Coppel RL, Stahl HD, Kemp DJ, Anders RF. Affinity purification of human antibodies directed against cloned antigens of Plasmodium falciparum. J Immunol Methods, 1986, 86, 257-264.

14 Hall R, Hyde JE, Goman M, Simmons DL, Hope IA, Mackay M, Scaife J, Merkli B, Rickle R, Stocker J. Major surface antigen gene of a human malaria parasite cloned and expressed in bacteria. Nature, 1984, 311, 379-382.

15 Perkins ME. Surface proteins of Plasmodium falciparum merozoites binding to the erythrocyte receptor, glycophorin. J Exp Med, 1984, 160, 788-798.

16 Ravetch JV, Kochan J, Perkins M. Isolation of the gene for a glycophorin-binding protein implicated in erythrocyte invasion by a malaria parasite. Science, 1985, 227, 1593-1597.

17 Wåhlin B, Wahlgren M, Perlmann H, Berzins K, Björkman A, Patarroyo ME, Perlmann P. Human antibodies to a Mr 155,000 Plasmodium falciparum antigen efficiently inhibit merozoite invasion. Proc Natl Acad Sci USA, 1984, 81, 7912-7916.

18 Coppel RL, Culvenor JG, Bianco AE, Crewther PM, Stahl HD, Brown GV, Anders RF, Kemp DJ. Variable antigen associated with the surface of erythrocytes infected with mature stages of Plasmodium falciparum. Molec Biochem Parasitol, 1986, 20, 265-277.

19 Leech JH, Barnwell JH, Miller LH, Howard RJ. Identification of a strain-specific malaria antigen exposed on the surface of Plasmodium falciparum - infected erythrocytes. $J$ Exp Med, 1984, 159, 1567-1575.

20 Holder AA, Freeman RR, Uni S, Aikawa M. Isolation of a Plasmodium falciparum rhoptry protein. Molec Biochem Parasitol, 1985, 14, 293-303.

21 Perrin LH, Merkli B, Loche M, Chizzolini C, Smart J, Richle R. Antimalarial immunity in Saimiri monkeys. Immunization with surface components of asexual blood stages. $J$ Exp Med, 1984, 160, 441-451.

22 Schofield L, Bushell GR, Cooper JA, Saul AJ, Upcroft JA, Kidson C. A rhoptry antigen of Plasmodium falciparum contains conserved and variable epitopes recognized by inhibitory monoclonal antibodies. Molec Biochem Parasitol, 1986, 18, 183-195.

23 Perrin LH, Ramirez E, Lambert PH, Miescher PA. Inhibition of $P$. falciparum growth in human erythrocytes by monoclonal antibodies. Nature, 1981, 289, 301-303. 
24 Schmidt-Ullrich R, Brown J, Whittle H, Lin P-S. Human-human hybridomas secreting monoclonal antibodies to the Mr 195,000 Plasmodium falciparum blood stage antigen. $J$ Exp Med, 1986, 163, 179-188.

25 Berzins K, Perlmann H, Wåhlin B, Carlsson J, Wahlgren M, Udomsangpetch R, Björkman A, Pattarroyo ME, Perlmann P. Rabbit and human antibodies to a repeated amino acid sequence of a Plasmodium falciparum antigen, Pf155, react with the native protein and inhibit merozoite invasion. Proc Natl Acad Sci USA, 1986, 83, 1065-1069.

26 Brown GV, Culvenor JG, Crewther PE, Bianco AE, Coppel RL, Saint RB, Stahl HD, Kemp DJ, Anders RF. Localization of the ring-infected erythrocyte surface antigen (RESA) of Plasmodium falciparum in merozoites and ring-infected erythrocytes. J Exp Med, 1985, 162, 774-779.

27 Perlmann H, Berzins K, Wahlgren M, Carlsson J, Björkman A, Patarroyo ME, Perlmann P. Antibodies in malarial sera to parasite antigens in the membrane of erythrocytes infected with early asexual stages of Plasmodium falciparum. J Exp Med, 1984, 159, 1686-1704.

28 Cowman AF, Coppel RL, Saint RB, Favaloro J, Crewther PE, Stahl H-D Bianco AE, Brown GV, Anders RF, Kemp DJ. The ring-infected erythrocyte surface antigen (RESA) polypeptide of Plasmodium falciparum contains two separate blocks of tandem repeats encoding antigenic epitopes that are naturally immunogenic in man. Molec Biol Med, 1984, 2, 207-222.

29 Zavala F, Tam JP, Hollingdale MR, Cochrane AH, Quakyi I, Nussenzweig RS, Nussenzweig V. Rationale for development of a synthetic vaccine against Plasmodium falciparum malaria. Science, 1985, 228, 1436-1440.

30 Cowman AF, Saint RB, Coppel RL, Brown GV, Anders RF, Kemp DJ. Conserved sequences flank variable tandem repeats in two S-antigen genes of P. falciparum. Cell, 1985, 40, 775-783.

31 Targett GAT, Sinden RE. Transmission blocking vaccines. Parasitology Today, 1985, 1, 155-158.

32 Anders RF. Multiple cross-reactivities amongst antigens of Plasmodium falciparum impair the development of protective immunity against malaria. Parasite Immunol, 1986 (in press). 